\title{
The Calpain Inhibitor A-705253 Attenuates Alcohol-Seeking and Relapse with Low Side-Effect Profile
}

\author{
Valentina Vengeliene', Achim Moeller², Marcus W Meinhardt', Patrick M Beardsley ${ }^{3}$, Wolfgang H Sommer', \\ Rainer Spanagel' and Anton Bespalov*,2,4 \\ IInstitute of Psychopharmacology, Central Institute of Mental Health, Faculty of Medicine Mannheim, University of Heidelberg, Mannheim, \\ Germany; ${ }^{2}$ Department of Neuroscience Research, AbbVie Deutschland GmbH \& Co. KG, Ludwigshafen, Germany; ${ }^{3}$ Department of Pharmacology \\ and Toxicology, School of Medicine, Virginia Commonwealth University, Richmond, VA, USA; ${ }^{4}$ Department of Psychopharmacology, Pavlov Medical \\ University, St Petersburg, Russia
}

\begin{abstract}
Preclinical studies revealed contribution of N-methyl-D-aspartate receptors (NMDARs) to a variety of neuropsychiatric diseases including alcoholism, but development of NMDAR antagonists for therapeutic use has been a challenge, in part due to severe side effects. One of the key intracellular events resulting from stimulation of NMDAR is activation of calpains - calcium-dependent cysteine proteases. Here we studied whether inhibition of calpains would produce therapeutic-like effects of NMDAR antagonists but without their NMDAR-mediated side-effect profile. The calpain inhibitor A-705253 (3-10 mg/ $\mathrm{kg}$ ) was tested in a model of cue-induced reinstatement of alcohol-seeking behavior in post-dependent Wistar rats and in an alcohol deprivation effect (ADE) model in long-term alcohol drinking Wistar rats, two behavioral models for alcohol-seeking and relapse, respectively. We also tested the effect of A-705253 on the saccharine deprivation effect (SDE) as a selectivity measure. Acute treatment with A-705253 dose-dependently reduced cue-induced reinstatement of alcohol-seeking behavior. Repeated administration of A-705253 caused significant reductions of relapse-like excessive alcohol intake during the postabstinence drinking days, an effect that persisted during two more successive drug-free drinking weeks, which was selective for the ADE as the SDE was unaffected. However, A-705253 did not produce psychostimulant, cognition impairing (delayed-matching-to-position), or psychotomimetic effects (specifically, phencyclidine discriminative stimulus effects). Taken together, these results demonstrate the involvement of calpains in alcohol-seeking and relapse and present a rationale for a novel pharmacological intervention that may reduce craving and relapse with minimal side effects in alcohol-dependent patients.

Neuropsychopharmacology (2016) 41, 979-988; doi:I0.1038/npp.2015.225; published online 16 September 2015
\end{abstract}

\section{INTRODUCTION}

Acute alcohol has a negative modulating effect on the N-methyl-D-aspartate receptor (NMDAR), whereas chronic alcohol use leads to the development of hypersensitivity of this receptor (Lovinger et al, 1989; Holmes et al, 2013). Together with augmented glutamate levels this causes hyperexcitability of the central nervous system during acute and conditioned withdrawal and contributes to alcohol-seeking and relapse (Herrmann et al, 2012; Holmes et al, 2013). On the basis of the role of NMDAR and glutamatergic system in alcohol-seeking and relapse, NMDAR antagonists have been suggested as a treatment of alcoholism (Krystal et al, 2003; Holmes et al, 2013; Spanagel and Vengeliene, 2013). However, clinical studies have so far not supported these expectations. In a pilot study in alcohol-dependent patients, treatment with the non-competitive NMDAR channel blocker, memantine, did not differentiate from placebo, but the investigators noted 'a

\footnotetext{
* Correspondence: Professor A Bespalov, Department of Neuroscience Research, AbbVie Deutschland $\mathrm{GmbH}$ \& Co. KG, Knollstreet 50, Ludwigshafen 67008, Germany, Tel: +49621 589 1370; Fax: +49 621 589 3232, E-mail: anton.bespalov@abbvie.com

Received 17 April 2015; revised 8 July 2015; accepted 9 July 2015; accepted article preview online 28 July 2015
}

greater number of side effects compared with the placebo group, such that $26 \%$ of patients had their drug dose decreased or discontinued due to memantine-related side effects' (Evans et al, 2007). Neramexane, another NMDAR channel blocker, also did not meet the primary outcome goals (continuous abstinence rate and cumulative abstinence duration) in a Phase II study in patients with alcohol dependence (Rammes, 2009; Spanagel and Vengeliene, 2013). Several reasons for the failure of these clinical studies have been discussed, but insufficient dosing might be the main reason (Spanagel and Vengeliene, 2013). However, given the severe psychotomimetic side-effect profile of high doses of NMDAR channel blockers that includes hallucinations, paranoid delusions, confusion, learning, and memory deficits, as well as many others, a conservative dosing regime in clinical trials is warranted.

There are several approaches one can take in order to maintain therapeutic efficacy while eliminating the adverse effects of NMDAR antagonists. One possible strategy is to identify intracellular signaling cascades coupled to NMDAR activation that are differentially involved in therapeutic $v s$ adverse effects of NMDAR antagonists. One of these intracellular signaling cascades involves calpains. Calpains are calcium-activated cysteine proteases with a crucial role in various pathophysiological processes in the cell (Goll et al, 
2003). Today, more than 15 mammalian calpains have been identified (Nimmrich et al, 2012). Predominantly, two isoforms of calpains, $\mu$-calpain (calpain I) and m-calpain (calpain II), exist in brain regions relevant for alcohol dependence (Witzmann et al, 2003; Goll et al, 2003; Noori et al, 2012). There are several pharmacological tools that can be used to selectively manipulate calpains to explore their role in physiology and pathology.

The present study aimed to evaluate effects of the brainpenetrating calpain I inhibitor A-705253 in models of alcoholseeking and relapse that were previously shown to be sensitive to NMDAR blockade-namely the model of cue-induced reinstatement of alcohol-seeking behavior in post-dependent Wistar rats (Meinhardt and Sommer, 2015) and the alcohol deprivation effect (ADE) model in long-term alcohol drinking Wistar rats (Spanagel and Hölter, 1999; Vengeliene et al, 2005). A-705253 has a Ki for calpain inhibition of $53 \mathrm{nM}$, and has been previously used in in vivo studies (Nimmrich et al, $2008)$, with a sufficiently long half-life $(2.3-5 \mathrm{~h})$ and, at $C_{\max }$, brain levels reach $90 \mathrm{ng} / \mathrm{g}(186 \mathrm{nM})$ after subcutaneous administration of $10 \mathrm{mg} / \mathrm{kg}$ in the rat (AbbVie, unpublished data; Lubisch et al, 2003).

The reinstatement model in post-dependent rat is used as a model for medication development in alcoholism (Meinhardt et al, 2013; Meinhardt and Sommer, 2015) and examines the resumption of responding in an operant task by re-exposing dependent and non-dependent rats to alcohol-associated environmental stimuli (cues; SanchisSegura and Spanagel, 2006). Cue-induced increase in alcohol-seeking is attenuated by some NMDAR antagonists (Bäckström and Hyytiä, 2004; Spanagel, 2009), but these effects are sometimes accompanied with a generalized impairment of motor behavior (Bachteler et al, 2005).

In the alcohol deprivation model in long-term alcohol drinking Wistar rats, renewed access to alcohol solutions after a period of deprivation for several days leads to a pronounced, although temporary, increase in voluntary alcohol intake (the ADE) in animals. Following repeated deprivation phases, the $\mathrm{ADE}$ is characterized by an increased demand for the drug that resembles a relapse situation in alcoholic patients (Spanagel and Hölter, 1999; Vengeliene et al, 2009). In previous studies, expression of ADE was attenuated by repeated sub-acute treatment with memantine, neramexane, and other NMDAR antagonists (Hölter et al, 2000; Vengeliene et al, 2005, 2008; Spanagel, 2009).

Here we performed: (i) dose-response testing of A-705253 in the reinstatement and ADE models; (ii) contrasted the observed effects $v s$ the saccharine deprivation effect (SDE) to see the selectivity of drug toward alcohol-related behaviors; and finally, (iii) assessed whether A-705253 would produce a NMDAR-like side-effect profile in a set of experiments aimed to reveal psychostimulant, psychotomimetic (phencyclidine-like), and cognition-impairing effects that are normally seen after the administration of NMDAR antagonists such as phencyclidine (PCP) or memantine.

\section{MATERIALS AND METHODS}

\section{Animals}

See Supplementary Information for details.

All experiments were approved by the AbbVie's Animal Welfare Office (Ludwigshafen, Germany) as well as institutional Committees on Animal Care and Use, and by the Regierungspräsidium Karlsruhe, and were performed in accordance with the European and German National Guidelines as well as the recommendations and policies of the United States National Institutes of Health Principles of Laboratory Animal Care. Except for the alcohol studies, all animal housing and experiments were conducted in facilities with full accreditation by the Association for Assessment and Accreditation of Laboratory Animal Care.

\section{Drugs}

See Supplementary Information for details.

\section{Cue-Induced Reinstatement of Alcohol-Seeking Behaviour in Post-Dependent Rats}

Operant alcohol self-administration apparatus. All alcohol-seeking experiments were carried out in operant chambers (MED Associates, St Albans, VT) enclosed in ventilated sound-attenuating cubicles. The chambers were equipped with a response lever on each side panel of the chamber (one active and another inactive). Responses at the active lever activated a syringe pump that delivered a $\sim 30-\mu 1$ drop of fluid into a liquid receptacle next to it. A light stimulus (house light) was mounted above the right response lever of the self-administration chamber. An IBM compatible computer controlled the delivery of fluids, presentation of stimuli, and data recording (see also Supplementary Information for details).

Alcohol self-administration training. All animal training and testing sessions were performed during the dark phase of their light cycle. Animals $(n=16)$ were trained to press the active lever reinforced with $10 \%(\mathrm{v} / \mathrm{v})$ ethanol in daily 30 min sessions using a fixed-ratio 1 (FR 1) schedule following Samson's sucrose-fading procedure (Samson, 1986). During the first 3 days of training, animals were kept fluid deprived for $20 \mathrm{~h}$ per day in order to facilitate acquisition of operant responding for a liquid reinforcer. Responses at the left lever were reinforced by the delivery of $0.2 \%(\mathrm{w} / \mathrm{v})$ saccharin solution. For the next 3 days, animals underwent the same procedure without fluid deprivation. Following the acquisition of saccharin-reinforced responding, rats were trained to self-administer ethanol. Thus, rats had access to $0.2 \%$ saccharin with $5 \%$ ethanol for 1 day, $5 \%$ ethanol for 1 day, $0.2 \%$ saccharin with $8 \%$ ethanol for 1 day, $8 \%$ ethanol for 1 day, $0.2 \%$ saccharin with $10 \%$ ethanol for 1 day, and $10 \%$ ethanol for 1 day. Throughout the training phase, responses at the inactive lever were recorded but not reinforced (inactive lever).

Conditioning phase. The purpose of the conditioning phase was to train the animals to associate the availability of ethanol with the presence of specific discriminative stimuli. This phase started after the completion of the saccharin-fading procedure. Discriminative stimuli predicting ethanol (10\%) availability were presented during each subsequent daily 30-min session. An orange flavor extract served as the cue stimulus ( $\mathrm{S}$ ) for ethanol. This olfactory stimulus was generated by depositing six drops of an orange extract into the bedding of the operant chamber before each 
session. In addition, each active lever press resulting in ethanol delivery was accompanied by a 5 -s presentation of the house light (conditioned light stimulus, CS). The 5-s period served as a 'time-out', during which responses were recorded but not reinforced. At the end of each session, the bedding of the chamber was changed and trays were thoroughly cleaned. The animals received a total of 10 ethanol conditioning sessions. Throughout the conditioning phase, responses at the inactive lever were recorded but not reinforced (inactive lever).

Ethanol vapor exposure and blood sampling. Vapor exposure experiment was performed in a rodent alcohol inhalation system as described previously (Rimondini et al, 2002). In this system, high-performance liquid chromatography pumps (Knauer, Berlin, Germany) deliver ethanol into electrically heated stainless steel coils $\left(60^{\circ} \mathrm{C}\right)$ connected to airflow of $18 \mathrm{l} / \mathrm{min}$. Vaporized ethanol is delivered to the individual rat chambers $\left(1 \times 1 \times 1 \mathrm{~m}^{3}\right)$ via the tubes connected to the side arms. Each tube also has its own pressure gauge that enables to adjust evenly the conditions for each chamber.

After completing the conditioning phase, rats were divided into two experimental groups, on the basis of their performance during the last four conditioning sessions $(n=8)$. One group was exposed to the ethanol vapor using an alcohol inhalation system while another group served as a control group. Control animals were placed into vapor chambers for the same amount of time without exposing them to ethanol vapor.

To induce dependence, rats first were allowed to habituate to the chambers for 1 week. Thereafter, rats were exposed to ethanol vapor for the following 7 weeks. Each week consisted of five 14-h (0000 hours to 1400 hours) vapor exposures separated by 10 -h periods of withdrawal and additional $58-\mathrm{h}$ of withdrawal at the end of the week.

Once per week blood $(\sim 20 \mu \mathrm{l})$ was sampled from the lateral tail vein of ethanol exposed rats for blood alcohol concentration measurements. Blood alcohol concentrations were determined using an AM1 Analox system (Analox Instruments, London, UK). Following intermittent exposure to ethanol vapor, all rats we subjected to the 2-week abstinence phase.

Conditioning and extinction phase in post-dependent rats. Following 2-week abstinence phase, all animals were re-conditioned to self-administer $10 \%$ ethanol in 10 daily conditioning sessions. After completing the re-conditioning phase, rats were subjected to daily 30 -min extinction sessions for 7 consecutive days, which in total were sufficient to reach the extinction criterion of less than 10 lever responses/ session. Extinction sessions began by extending the levers without presenting olfactory discriminative stimuli. Responses at the previously active lever activated the syringe pump, without resulting in the delivery of ethanol or the presentation of response-contingent cues (stimulus light).

Reinstatement testing. Reinstatement tests began 3 days after the final extinction session. In these tests, rats were exposed to the same conditions as during the conditioning phase, except that the ethanol was not made available. Sessions were initiated by the extension of both ethanol associated and inactive levers and the presentation of the discriminative stimulus predicting ethanol (S). Responses at the ethanol-associated lever were followed by the activation of the syringe pump and the presentation of the CS (light). The first two lever presses resulted in the delivery of ethanol, ie, $\sim 60 \mu$ of liquid that served as an additional olfactory/ gustatory cue (see also Vengeliene et al, 2007, for details).

To test the effect of A-705253 on cue-induced reinstatement, animals were injected IP with either vehicle, $3 \mathrm{mg} / \mathrm{kg}$ of A-705253, or $10 \mathrm{mg} / \mathrm{kg}$ of A-705253 randomized throughout three reinstatement sessions separated by 5 days based on a Latin square design. A single-drug administration was performed $30 \mathrm{~min}$ before each reinstatement session. (see Supplementary Information for locomotor activity measurements).

\section{Long-Term Voluntary Alcohol Consumption with Repeated Deprivation Phases}

Alcohol consumption with repeated deprivation phases. After 2 weeks of habituation to the animal room, rats $(n=24)$ were given ad libitum access to tap water and to 5, 10 , and $20 \%$ ethanol solutions (v/v) as well. Spillage and evaporation were minimized by the use of special bottle caps. With this procedure, the ethanol concentration remains constant for at least 1 week (Hölter et al, 1998). The positions of bottles were changed weekly.

The first 2-week deprivation period was introduced after 8 weeks of continuous alcohol availability. After the deprivation period, rats were given access to alcohol again and three more deprivation periods were introduced in a random manner, ie, the duration of following drinking and deprivation phases was irregular, ie, $\sim 5 \pm 1$ weeks and $2 \pm 1$ weeks, respectively, to prevent adaptive behavioral mechanisms (see also Vengeliene et al, 2005, 2010, for details). The long-term voluntary alcohol drinking procedure including all deprivation phases lasted a total of 8 months.

The pharmacological testing was introduced at the end of the fourth alcohol deprivation period. To study the effects of A-705253, rats were divided into three groups $(n=8)$ in such way that the mean baseline total alcohol intake was approximately the same in each group (ie, $\sim 1.9 \mathrm{~g} / \mathrm{kg} / \mathrm{day}$ ). Baseline drinking was measured daily for 1 week. After the last day of baseline measurement, the alcohol bottles were removed from the cages leaving the animals with free access to food and water for 2 weeks. Thereafter, each animal was subjected to a total of 5 IP injections (starting at 1900 hours with $12 \mathrm{~h}$ intervals) of either vehicle or A-705253 (3 and $10 \mathrm{mg} / \mathrm{kg}$ ). The alcohol bottles were reintroduced after the second injection (at $\sim 0900$ hours on the 15th day of alcohol deprivation) and the occurrence of an ADE was determined. Total ethanol ( $\mathrm{g} / \mathrm{kg}$ of body weight/day) and water intake ( $\mathrm{ml} / \mathrm{kg}$ of body weight/day) were measured daily at $\sim 0900$ hours for the subsequent week. To test for persistent treatment effects, both ethanol and water intake were measured for three more weeks, and one more drug-free ADE. Each rat's body weight was recorded $24 \mathrm{~h}$ before the first injection and $12 \mathrm{~h}$ after the last injection. The drug injection schedule was based on our previous studies using the same paradigm (eg, see Vengeliene et al 2010, for details). In addition, home cage locomotor activity was measured by the E-motion system (see Supplementary Information for details). 


\section{Saccharine Deprivation Effect}

After 2 weeks of habituation to the animal room, rats were given ad libitum access to tap water and $0.1 \%$ saccharine solution (w/v) as well. The positions of bottles were changed weekly. A 1-week deprivation period was introduced after 18 weeks of continuous saccharin availability. After the deprivation period, rats were given access to saccharin again.

The pharmacological testing was performed in the same way as in the above described ADE studies. Briefly, drug administration started at the end of the saccharine deprivation period. To study the effects of A-705253, rats were divided into two groups $(n=8)$ in such way that the mean baseline saccharine intake was approximately the same in each group (ie, $\sim 29 \mathrm{ml} /$ day). After the last day of baseline measurement, the saccharine bottles were removed from the cages leaving the animals with free access to food and water for 1 week. At the end of this week, each animal was subjected to a total of 5 IP injections (starting at 1900 hours with $12 \mathrm{~h}$ intervals) of either vehicle or A-705253 $(10 \mathrm{mg} / \mathrm{kg})$. The saccharine bottles were reintroduced after the second injection and the occurrence of SDE was determined.

\section{Assessment of Side-Effect Profile}

Locomotor activity test. Thirty minutes after administration of either vehicle or A-705253 (3, 10 and $30 \mathrm{mg} / \mathrm{kg}$ ), animals ( $n=8$ per treatment condition) were put in a Type IV Macrolon test cage with bedding and a filter-unit lid, which was placed in a frame with photobeams (Cage Rack Photobeam system, San Diego Instruments, San Diego, CA) housed inside the sound attenuating cubicles (Med Associates) with house lights on (20-25 Lux) and a small ventilator fixed on the wall of the cubicle to generate ventilation and background noise. After breaking the first light barrier, the monitoring of locomotion was started and activity was recorded for $2 \mathrm{~h}$.

PCP discrimination testing. Drug discrimination training and test sessions were conducted in two-lever operant conditioning chambers (MED Associates) equipped with a house light and a food dispenser that delivered $45 \mathrm{mg}$ food pellets (P.J. Noyes Company, Frenchtown, NJ). Scheduling of pellet deliveries and collection of data were accomplished by a microcomputer, interface, and associated software (MED-PC, MED Associates).

Subjects $(n=7)$ were initially trained to press one of the two levers under a fixed ratio 1 (FR1) schedule of food reinforcement in which a food pellet was delivered following each press of a designated lever. The response requirement was gradually increased to FR10. Subsequently, the rats were reinforced for pressing the opposite lever until FR10 performance was established. Drug-discrimination training then began during daily, 15-min experimental sessions. At the start of each session, a white house light was turned on, and in its presence the rats were required to make 10 consecutive responses on the lever appropriate to the presession treatment to obtain food pellet delivery. Rats were injected i.p. $15 \mathrm{~min}$ before each session with $2 \mathrm{mg} / \mathrm{kg}$ of PCP or saline. One of the two levers was designated as the 'correct lever' dependent upon whether PCP or saline was administered. PCP and saline injections were scheduled according to a double alternating sequence. Lever pressing produced pellet delivery only on the injection-appropriate lever for that day. Incorrect presses reset the response requirement on the correct lever during training sessions (but not during test sessions; see below).

Testing began once a subject met the following criteria: (i) the first completed fixed-ratio occurred on the lever designated correct on at least 8 of 10 consecutive training sessions and (ii) at least $80 \%$ of the total responses were made on the correct lever during those 8 sessions. After these initial training conditions were met, tests could occur on Tuesdays and Fridays provided that the subject completed the first completed fixed-ratio on the correct lever during the most recent training drug and vehicle sessions, otherwise a training session was administered. Test days were identical to training days except completions of the fixed ratio contingencies at either lever resulted in pellet delivery. A dose-response curve was initially obtained for PCP $(0.5-8 \mathrm{mg} / \mathrm{kg})$ followed by substitution tests with A-705253 $(3,10$, and $30 \mathrm{mg} / \mathrm{kg})$ and its vehicle.

Delayed non-matching to position (DNMTP) test. Experiments were conducted in standard operant conditioning chambers (MED Associates, East Fairfield, VT) enclosed in sound- and light-attenuating cubicles. The chambers were connected to a computer through an interface and controlled by custom-programmed MED-PC scripts. Each chamber was equipped with a white house light centered $19 \mathrm{~cm}$ above two response levers (model ENV-112BM; positioned $7 \mathrm{~cm}$ above the floor), and a food dispenser, which delivered $45 \mathrm{mg}$ food pellets (Formula A/I, Noyes Precision Pellets, Research Diets, Inc., New Brunswick, NJ) on the front wall. Two cue lights were positioned above each lever, and an additional light illuminated the food tray. A nose-poke operandum with the built-in cue light (model ENV-114M) was situated on the back side of the chamber.

First, rats $(n=12$ for experiments with memantine and $n=9$ in studies on A-705253) were shaped to lever press for food pellet delivery and then were taken through several consecutive training stages as described earlier (Sukhotina et al, 2008). All animals were trained in parallel and needed about 50 sessions to achieve the final stage of the training. Start of each trial was signaled by the illumination of the house light and with one lever extension into the chamber (the 'sample'). If the rat pressed the lever, the lever was retracted and the food magazine was illuminated. After a variable delay period $(0,8,16$, or $32 \mathrm{~s})$, the cue light inside the nose-poke hole was illuminated and the rat had to nosepoke in order to get both levers presented for making a choice. If the animal pressed the lever opposite to the 'sample' (ie, non-matching), then the correct response was recorded and both levers were retracted, and the rat was reinforced with a food pellet. If the rat pressed the same lever as the 'sample' (ie, matching), then an incorrect response was recorded, both levers were retracted, and no food was delivered. Each trial was followed by the inter-trial interval of $5 \mathrm{~s}$ with the house light off. Each session consisted of 96 trails. During each trial, the rat had $20 \mathrm{~s}$ to respond to the 'sample' lever. If no lever was pressed during this period, it was retracted and the house light was extinguished for $20 \mathrm{~s}$ ('time-out' period). Failure to respond upon a lever was recorded as a missed trial or omission. This was followed by 
an inter-trial interval of $5 \mathrm{~s}$ with the house light off. Training continued until the animals reached the criteria of: (i) $90 \%$ correct at 0-s delay, (ii) $40-60 \%$ correct at the longest delay, (iii) not more than 5 omissions, and (iv) $40-60 \%$ left-hand side preference. A session lasted about 40-50 $\mathrm{min}$.

\section{Statistics}

Data obtained from the cue-induced reinstatement experiment were analyzed by use of a two-way analysis of variance (ANOVA) with repeated measures (factors were: treatment and lever). Locomotor activity measurements for the reinstatement experiment were analyzed using one-way ANOVA with repeated measures (factor-treatment). Whenever significant differences were found, post-hoc Student-Newman-Keuls tests were performed.

Data derived from home-cage drinking (total alcohol intake and water intake) and home-cage locomotor activity were analyzed using a two-way ANOVA with repeated measures (factors were: treatment and day/week). Data analysis regarding the effects of treatment on the change in the animals' body weight was performed using a one-way ANOVA (factortreatment). Whenever significant differences were found, post-hoc Student-Newman-Keuls tests were performed.

For locomotor activity test, cumulative activity counts (over 2-h sessions) were subjected to one-way ANOVA and post-hoc comparisons.

In drug discrimination studies, the percent drug-lever responding was calculated for each subject by dividing the number of lever presses emitted upon the PCP-designated lever by the total number of lever presses emitted upon both levers and multiplying this quotient by 100. Individual drug lever responding percentages were then averaged $( \pm$ SEM). Complete generalization to the PCP discriminative stimulus was inferred when \% PCP lever responses $\geqslant 80 \%$. Mean response rates for each test condition were calculated by dividing the total number of lever presses emitted upon both levers by the duration of the test session ( $900 \mathrm{~s}$ ) for each subject and then these rates were averaged $( \pm$ SEM). Data were subjected to repeated measures ANOVA, and results at each drug dose condition were compared with results obtained under vehicle-control conditions with post-hoc testing. If a rat failed to emit at least 10 lever presses (ie, sufficient to obtain at least one pellet delivery) during a test, its data were excluded from calculations of mean \%PCP lever responding for that test but were included for mean response rate determinations. This exclusion was made to prevent disproportionate influence on expressions of PCP-lever selection by extremely low rate responding rats. ED50's were determined via curvilinear analysis.

For DNMTP, percent of correct responses (choice accuracy) was calculated for each individual rat at each delay interval and, after rank transformation, subjected to a two-way ANOVA with repeated measures on both factors (treatment and delays). Whenever applicable for repeated measures analysis, Mauchly's test of sphericity was applied and the degrees of freedom were corrected to more conservative values using the Huynh-Feldt's epsilon for any terms involving factors in which the sphericity assumption was violated.

Differences during all comparisons were inferred when $P<0.05$.

\section{RESULTS}

\section{A-705253 Dose-Dependently Reduces Cue-Induced Reinstatement of Alcohol-Seeking Behavior in Post- Dependent Rats}

At the end of the second conditioning phase, non-dependent rats exhibited $56 \pm 4$ ethanol reinforced lever presses, whereas post-dependent rats had $125 \pm 7$ lever presses. The number of operant responses progressively diminished across seven extinction sessions. Thus, during the last extinction session, lever presses were as low as $5.8 \pm 1.5$ and $7.8 \pm 0.4$ for the control and post-dependent group, respectively.

During the reinstatement test, both animal groups (non-dependent and post-dependent) increased the number of responses on the ethanol-associated lever as compared with the last extinction session. This was confirmed by the repeated measures ANOVA results (factor treatment: $\mathrm{F}(3,21)=19.1, P<0.0001$ and $\mathrm{F}(3,21)=21.6, P<0.0001$ for the non-dependent animal group and post-dependent group, respectively). Post-hoc comparisons revealed that responses on the ethanol-associated lever during the reinstatement test were increased significantly in vehicle as well as the $3 \mathrm{mg} / \mathrm{kg}$ of A-705253-treated groups (Figure 1). However, treatment with $3 \mathrm{mg} / \mathrm{kg}$ of A-705253 reduced lever responses significantly during the reinstatement testing in both nondependent and post-dependent animal groups, whereas $10 \mathrm{mg} / \mathrm{kg}$ dose of A-705253 completely abolished cueinduced enhanced ethanol-seeking (Figure $1 \mathrm{a}$ and c). A-705253 treatment did not affect responding at the inactive lever (Figure $1 \mathrm{~b}$ and $\mathrm{d}$ ). Locomotor activity measurements showed a slight, although not significant, reduction in animals' activity following administration of both 3 and $10 \mathrm{mg} / \mathrm{kg}$ of A-705253 (factor treatment: $P=0.55$ ). Number of movements during the $30 \mathrm{~min}$ that corresponded to the time of reinstatement testing for the vehicle control group was $504 \pm 50$, for the $3 \mathrm{mg} / \mathrm{kg}$ treatment group was $432 \pm 53$, and for the $10 \mathrm{mg} / \mathrm{kg}$ treatment group was $409 \pm 77$.

\section{A-705253 Abolishes the ADE but not the SDE}

Following the re-introduction of alcohol solutions after a period of abstinence, the vehicle-treated group showed the expected increase in alcohol consumption, indicating occurrence of an $\mathrm{ADE}$ (Figure 2). With respect to the pharmacological treatments, a two-way ANOVA for repeated measures revealed a significant increase in alcohol intake after a deprivation phase in all animal groups as compared with basal drinking (factor day: $F(7,168)=38.1$, $P<0.0001)$. This increase was mainly attributable to the vehicle-treated group, whereas A-705253 treatment reduced the expression of ADE. In A-705253-treated animals, alcohol intake was still increased during the first post-deprivation day but dropped below baseline levels from the second day onward. Thus, two-way ANOVA revealed a significant difference in alcohol intake between vehicle- and A-705253treated animal groups (factor treatment group: $F(2,21)=$ 22.4, $P<0.0001)$ and a significant treatment group $\times$ day interaction effect $(\mathrm{F}(14,147)=6.1, P<0.0001)$, showing that the treatment of rats with A-705253 was capable of reducing the expression of ADE (Figure 2). In contrast, water intake (Supplementary Figure 1) was significantly increased in A-705253-treated animals during treatment days, suggesting 

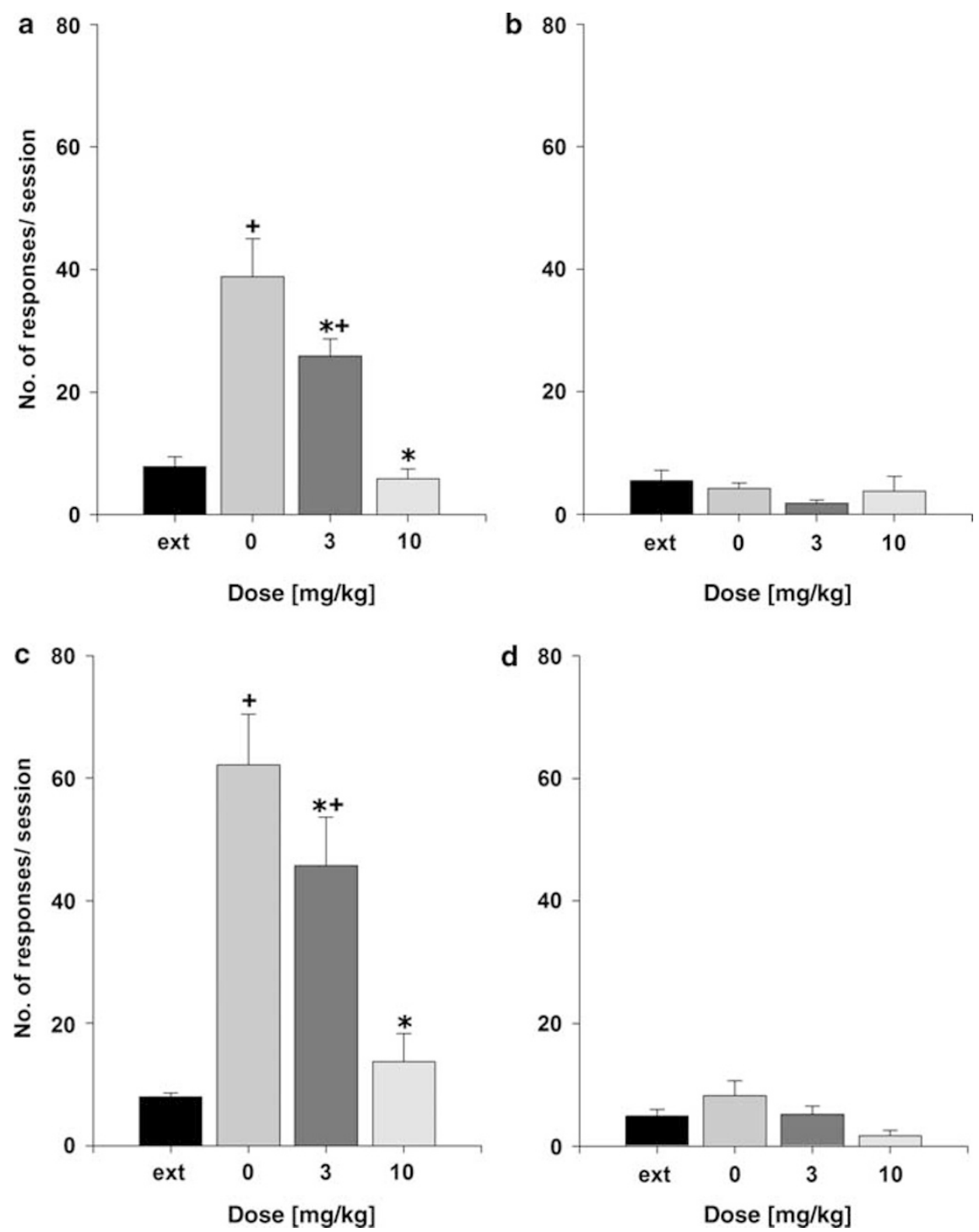

Figure I The effect of vehicle $(0 \mathrm{mg} / \mathrm{kg}$ ), $3 \mathrm{mg} / \mathrm{kg}$ of A-705253, and $10 \mathrm{mg} / \mathrm{kg}$ of A-705253 ( $n=8$ per-treatment condition) on ethanol cue-induced reinstatement in $(a, b)$ non-dependent and $(c, d)$ post-dependent rats. Data are shown as the average number of lever presses on ethanol-associated lever during the last four extinction sessions (ext) and as the number of responses after the presentation of stimuli previously paired with ethanol (a, $c$ ) and as the number of responses on the inactive lever $(b, d)$. Data are presented as means \pm SEM. ${ }^{+}$Significant differences to the extinction lever responses; * ${ }^{*}$ ignificant differences from the vehicle control group, $P<0.05$.

that the effect of treatment was selective for alcohol (factor treatment group $\times$ day interaction effect $(\mathrm{F}(14,147)=2.2$, $P<0.05)$ ). It should also be noted that the $10 \mathrm{mg} / \mathrm{kg}$ dose of A-705253 treatment led to small but significant changes in the animals' body weight (factor treatment group: $\mathrm{F}(2,21)=$ 22.4, $P<0.0001$; difference in body weight when compared before and after treatment: $+0.2 \pm 0.2 \%, 3 \mathrm{mg} / \mathrm{kg}$ treated animals: $-0.2 \pm 0.2 \%$ and $10 \mathrm{mg} / \mathrm{kg}$ treated animals: $-1.8 \pm 0.3 \%$ ). This small reduction in body weight is most likely due to reduced alcohol intake.

Monitoring of weekly ethanol intake after A-705253 treatment showed that alcohol consumption was significantly different among the three treatment groups over the entire time-course of the experiment (factor treatment group: $\mathrm{F}(2,21)=7.0, P<0.01$ and treatment group $\times$ week interaction effect: $F(16,168)=2.7, P<0.001$; Supplementary Figure 2). Subsequent post-hoc analysis revealed that alcohol consumption in the $10 \mathrm{mg} / \mathrm{kg}$ of A-705253 treatment group was significantly reduced during two more successive drug-free drinking weeks as compared with the vehicle-treated group.

Locomotor activity data for the ADE experiment were analyzed using recordings of $12 \mathrm{~h}$ post-injection intervals that corresponded to the animals' active phase. Overall, there was a general reduction in home-cage activity seen in all animal groups, which was likely caused by alcohol intoxication. A two-way ANOVA revealed a significant difference in activity of A-705253-treated animals, when compared with the vehicle-treated rats (factor treatment group $\times$ day interaction effect: $\mathrm{F}(14,147)=1.9, P<0.05$; Supplementary Figure 3). Post-hoc analysis revealed that locomotor activity in the $3 \mathrm{mg} / \mathrm{kg}$ treated animal groups was not significantly different from the vehicle-treated animal group at any time point. However, repeated administration of $10 \mathrm{mg} / \mathrm{kg}$ of A-705253 
caused a slight reduction of locomotor activity, only to recover to basal levels immediately after treatment stopped.

To test the selectivity of A-705253 toward alcohol-related effects, we studied the occurrence of a SDE. Following the re-introduction of saccharin after a period of abstinence, its consumption increased in both vehicle- and A-705253treated subjects as compared with baseline saccharin intake, indicating occurrence of an SDE. This was confirmed with a two-way ANOVA for repeated measures (factor day: $\mathrm{F}(3,87)=5.9, P<0.001)$. However, A-705253 treatment did not reduce the expression of SDE (factor treatment group:

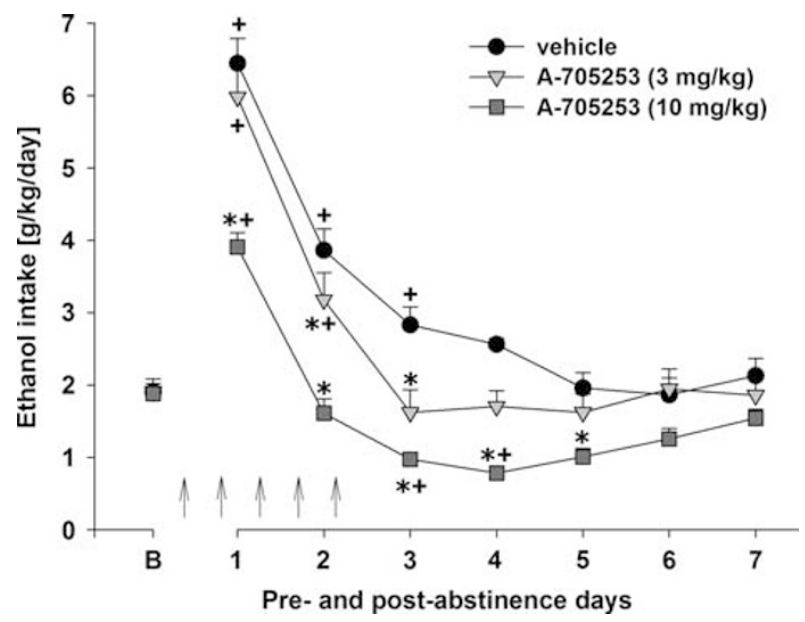

Figure 2 Intake of total ethanol (calculated in g of pure alcohol per $\mathrm{kg}$ of body weight per day) before and after a deprivation period of 2 weeks. The last 3 days measurements of ethanol intake is given as baseline drinking - 'B'. Arrows indicate the administration of either vehicle, $3 \mathrm{mg} / \mathrm{kg}$ of A-705253, or $10 \mathrm{mg} / \mathrm{kg}$ of $\mathrm{A}-705253$ ( $n=8$ per treatment condition). Data are presented as means \pm SEM. 'Significant differences to baseline drinking, *significant differences from the vehicle control group, $P<0.05$.
$P=0.71$ and factor treatment group $\times$ day interaction effect: $P=0.31$; Supplementary Figure 4).

\section{A-705253 does not Produce a Typical NMDAR-Mediated Side-Effect Profile}

Application of NMDAR antagonists can produce psychomotor stimulation. Indeed locomotor activity was significantly enhanced by pretreatment with different doses $(0,2.5,5$, and $10 \mathrm{mg} / \mathrm{kg})$ of the channel blocker memantine $(\mathrm{F}(3,31)=$ 9.7, $P<0.01$ ), whereas only a very high dose of A-705253 $(30 \mathrm{mg} / \mathrm{kg}$ ) - which was not used for the alcohol-seeking and relapse experiments-reduced motor activity $(\mathrm{F}(3,31)=5.5$, $P<0.01$; Figure 3).

Another spectrum of NMDAR antagonistic side effects is related to dissociative, hallucinogenic, and euphoriant properties. This can best be seen after the application of PCP. We therefore asked whether A-705253 is able to substitute for PCP in a discrimination task. When PCP was tested, it dose-dependently engendered the $2 \mathrm{mg} / \mathrm{kg}$ PCP discriminative stimulus with an $\mathrm{ED}_{50}$ of 1.11 (CI: $\left.0.85-1.44\right) \mathrm{mg} / \mathrm{kg}$ (Figure 4a). When A-705253 was tested, it produced maximum \% PCP lever presses of $10.2 \pm 6.2 \%$, indicating that it did not substitute to the PCP discriminative stimulus at the doses tested. Both PCP and A-705253 produced dosedependent reductions in response rates, and had $\mathrm{ED}_{50} \mathrm{~S}$ of 3.86 (CI: $2.88-5.18) \mathrm{mg} / \mathrm{kg}$ and 11.59 (CI: $2.21-60.67) \mathrm{mg} / \mathrm{kg}$ for doing so, respectively (Figure $4 \mathrm{~b}$ ). These response rate effects of A-705253 indicated that it was tested at doses high enough to produce behavioral effects.

NMDAR blockers such as memantine can also produce cognition-impairing effects (Creeley et al, 2006; Holmes et al, 2013). One test that is especially sensitive to cognitionimpairing effects is the DNMPT test (Koch et al, 2015). By the end of DNMTP training, rats demonstrated stable performance that depended on the delay duration. Under no-drug conditions, performance accuracy was almost $100 \%$
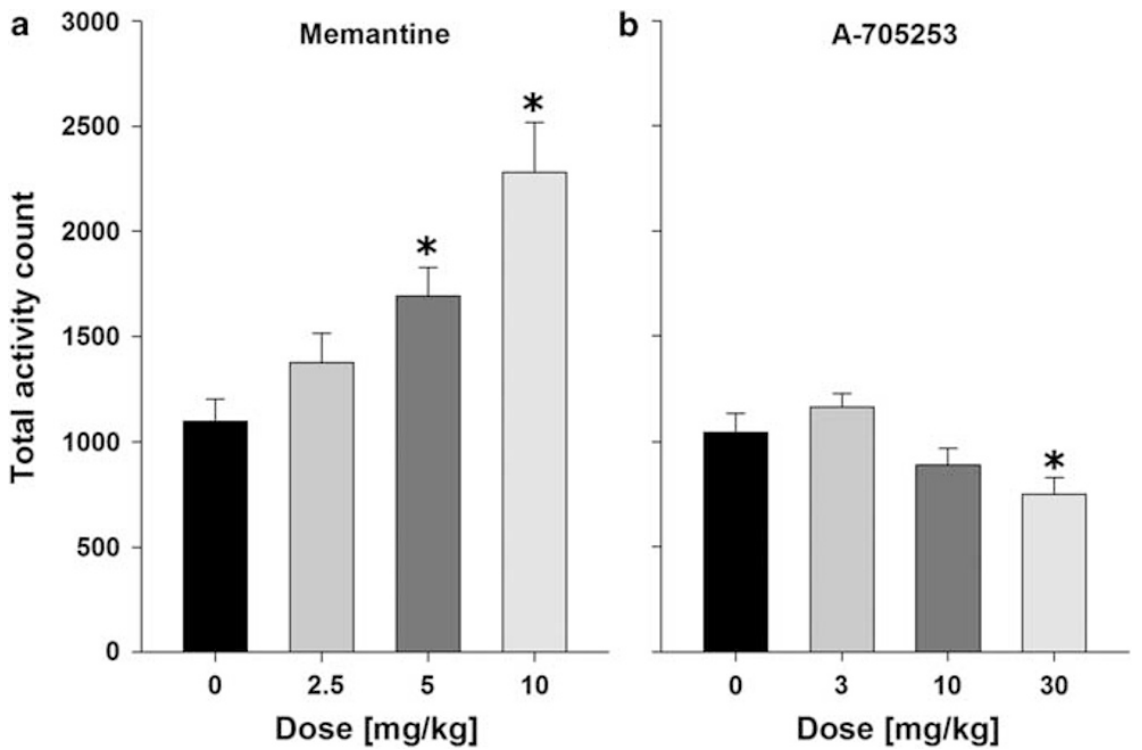

Figure 3 Effects of memantine (a) and A-705253 (b) on locomotor activity. Thirty minutes after the drug injection, rats ( $n=8$ per treatment condition) were placed into test cages and their activity was recorded for $2 \mathrm{~h}$. Data are presented as means $\pm \mathrm{SEM}$. *Significant differences from the corresponding vehicle control group, $P<0.05$. 
at zero delay level and approached $50 \%$ chance level at the longest delay level. Such delay dependence of DNMTP performance was maintained throughout the rest of the experiment during training sessions as well as test sessions conducted after vehicle administration. Pretreatment with memantine had profound effects on DNMTP performance
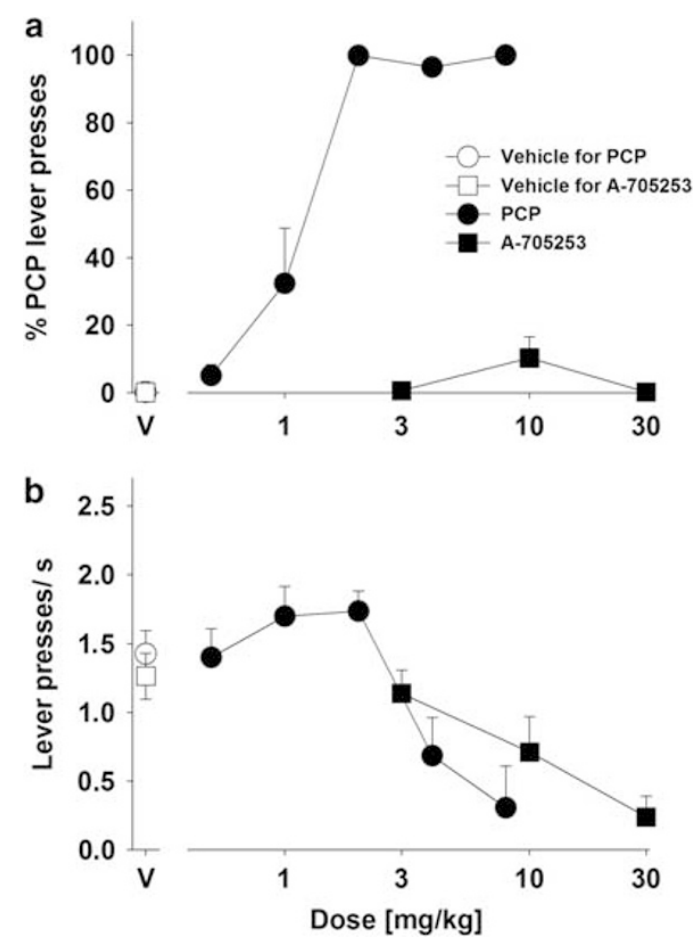

Figure 4 Results of substitution tests with phencyclidine (PCP), A-705253, and their vehicles (V) that were administered to rats trained to discriminate $2 \mathrm{mg} / \mathrm{kg}$ PCP from saline. (a) Effects on \%PCP lever presses. Each data point represents the mean $( \pm$ SEM) percentage of presses of the PCP-associated lever relative to presses of both levers, $n=7$ (note: six rats at $8 \mathrm{mg} / \mathrm{kg}$ of PCP and four rats at $30 \mathrm{mg} / \mathrm{kg}$ of A-705253 were excluded because of low response rates). (b) Effects on response rates (lever presses/s). Each data point represents the mean $( \pm$ SEM) total number of lever presses per second during the 15 min test session $(n=7)$. reducing the overall accuracy (Figure 5a; $\mathrm{F}(1,66)=9.6$, $P<0.01)$. These effects were associated with a significant increase in choice latencies (two-tailed paired $t$-test, $t=2.6$, $\mathrm{df}=12, P<0.05)$ and a trend toward a higher number of trials omitted (Supplementary Table 1). In contrast, up to the highest tested dose of $10 \mathrm{mg} / \mathrm{kg}, \mathrm{A}-705253 \mathrm{had}$ no impact on any of the recorded parameters (Figure $5 \mathrm{~b}$ and Supplementary Table 1).

\section{DISCUSSION}

The present study is the first to demonstrate that calpain inhibition attenuates behaviors related to alcohol-seeking and relapse in laboratory rats. Acute treatment with A-705253 reduced cue-induced reinstatement of alcohol-seeking behavior, whereas repeated administration of A-705253 caused significant reductions of relapse-like excessive alcohol intake during the post-abstinence drinking days in a four-bottle free choice paradigm. This treatment effect was observed during two more successive drug-free drinking weeks and was selective for the $\mathrm{ADE}$, as the SDE was not affected by subchronic A-705253 treatment. Thus, sub-chronic treatment with A-705253 may also induce long-lasting changes in the brain activity, which affects excessive drinking for several weeks after treatment cessation. Most important, calpain inhibition seems to generate a very low side-effect profile, which is devoid of cognition impairment, psychomotor stimulation, or any other psychotomimetic effects.

Previous laboratory research has revealed calpain activation after exposure to alcohol (Rajgopal and Vemuri, 2002; Carloni et al, 2004; Cho et al, 2014). Besides calpain activation induced by exposure to alcohol, there is also evidence of higher expression of calpain in alcohol-naïve rats of an alcohol-preferring strain (Witzmann et al, 2003). Taken together with the results of the present study showing that calpain inhibition attenuates alcohol-seeking and alcohol taking behaviors, higher expression of calpain found by Witzmann and colleagues may indicate that higher calpain activity might underlie preference for alcohol, at least in certain subjects or strains.

Calpains are often discussed as one of the intracellular targets mediating at least some of the effects of NMDA
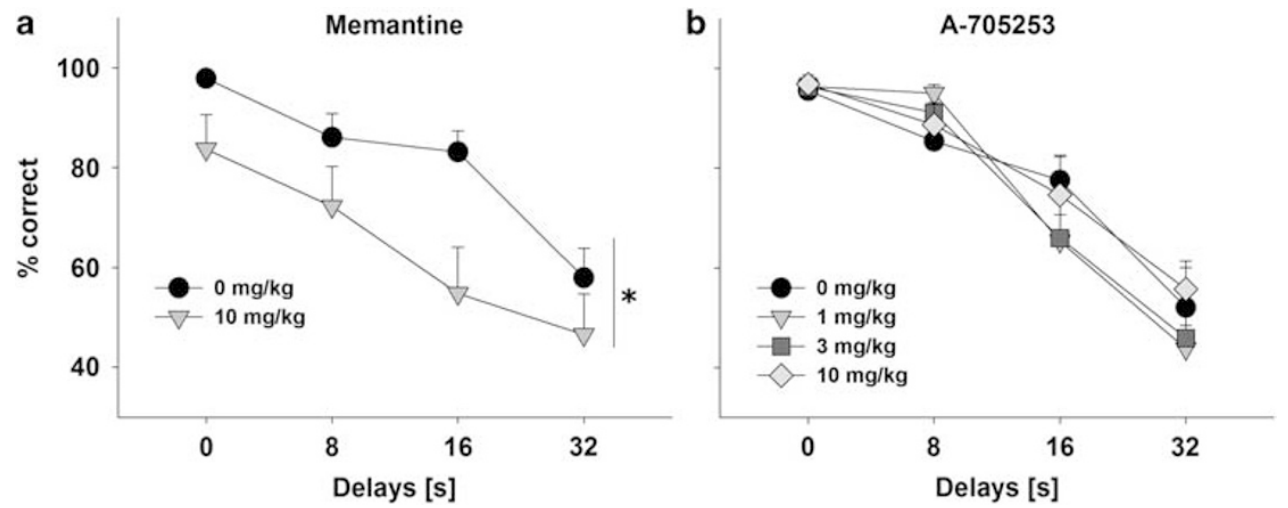

Figure 5 Effect of (a) memantine and (b) A-705253 on the accuracy of performance in the delayed non-matching to position (DNMTP) test. Memantine $(10 \mathrm{mg} / \mathrm{kg}), \mathrm{A}-705253(\mathrm{I}-10 \mathrm{mg} / \mathrm{kg})$, or their respective vehicle $(n=9-12$ per treatment condition) were injected 30 min before the test. Data are presented as mean $( \pm$ SEM) percentage of correct lever choice (maximum of 24 choices per delay per session), $* P<0.05$, main effect of treatment. 
receptor activation (Goll et al, 2003). Not unexpectedly, very similar to NDMAR antagonists (Nyakas et al, 2011), calpain inhibitors exert neuroprotective effects in an animal model (Nimmrich et al, 2008). The present study suggests that calpain inhibitors may share other therapeutically interesting effects described previously for NMDAR antagonists.

In our earlier studies, we demonstrated that repeated administration of either competitive or uncompetitive NMDAR antagonists in rats dose-dependently suppressed alcohol consumption during relapse-like situations, demonstrating that inhibition of NMDAR activity is effective for reducing relapse-like drinking behavior (Hölter et al, 2000; Vengeliene et al, 2005, 2008; Spanagel, 2009; Holmes et al, 2013). However, NMDAR antagonists produce phencyclidine- or alcohol-like discriminative stimulus effects (Hundt et al, 1998; Kostowski and Bieńkowski, 1999), stimulate motor activity (Danysz et al, 1994), produce cognitionimpairing effects (Creeley et al, 2006; Holmes et al, 2013), and have abuse potential (Holmes et al; 2013). In contrast, calpain inhibition affects only one of the signaling pathways activated by stimulation of NMDARs, but it, nevertheless, attenuated alcohol-seeking and relapse-like drinking in the absence of any side effects commonly reported for competitive and non-competitive NMDAR antagonists-locomotor stimulation, memory impairment, or substitution for PCP in a drug discrimination paradigm.

Since the 1980s, there were numerous studies that revealed therapeutic potential of NMDAR antagonists but these hopes largely failed to translate into any medication approved for clinical use. The main obstacle was and still remains to be side effects associated with NMDAR blockade (Danysz et al, 1994; Krystal et al, 1999; Holmes et al, 2013). One possible path forward to solve this problem is identification of processes downstream to NMDARs that would be able to retain therapeutic efficacy and not the side effects. Adding to the previously published evidence on neuroprotective effects of calpain inhibition (Nimmrich et al, 2008), the present study further points at calpain inhibition as the strategy to selectively retain therapeutic benefits of NMDAR antagonists.

However, as attractive as this hypothesis may sound, it should be noted that calpains are calcium-activated cysteine proteases and several mechanisms other than NMDARs modulate intracellular $\mathrm{Ca}^{2+}$ levels and calpain activation (eg, $\mathrm{Ca}^{2+}$ derived from endoplasmic reticulum stores or via voltage-activated calcium channels). In other words, calpain inhibitors may have a number of qualities that are not shared by NMDAR antagonists. For example, it may be worth noting that NMDAR antagonists attenuate both ADE and SDE (Zakharova et al, 2004), whereas our results indicate that A-705253 had no impact on SDE. Further, NMDAR antagonists do not reliably reduce conditioned alcoholseeking responses (Bäckström and Hyytiä, 2004; Bachteler et al, 2005; Spanagel, 2009) and in some cases significant reduction of these behaviors occurs only at the doses which impair motor coordination of the animals (Bachteler et al, 2005) or at the doses that produce alcohol-like subjective effects in humans (Krupitsky et al, 2007). In contrast, in the present study, we demonstrated that administration of calpain inhibitor A-705253 reduced alcohol cue-induced reinstatement in both non-dependent and post-dependent rats without producing significant reduction of responding at the inactive lever or the locomotor activity of the animal.
In summary, the present results show that calpain inhibition may selectively reduce cue-induced reinstatement of ethanol-seeking behavior and ADE, animal models previously argued to have predictive validity for treatment development (Shaham et al, 2003; Sanchis-Segura and Spanagel, 2006; Vengeliene et al, 2008; Meinhardt and Sommer, 2015). Therefore, these data present a rationale for a novel pharmacological intervention that may reduce craving and relapse in alcohol-dependent patients.

\section{FUNDING AND DISCLOSURE}

AbbVie participated in the design and conduct of the study, interpretation of data, review, and approval of the publication. $\mathrm{AB}$ and $\mathrm{AM}$ are $\mathrm{AbbVie}$ employees. Financial support for this work was provided by AbbVie and by the Bundesministerium für Bildung und Forschung (e:Med program, FKZ: 01ZX1311A (Spanagel et al, 2013)). The authors declare no conflict of interest.

\section{ACKNOWLEDGMENTS}

We thank Sabrina Koch for the excellent technical assistance.

\section{REFERENCES}

Bachteler D, Economidou D, Danysz W, Ciccocioppo R, Spanagel R (2005). The effects of acamprosate and neramexane on cue-induced reinstatement of ethanol-seeking behavior in rat. Neuropsychopharmacology 30: 1104-1110.

Bäckström P, Hyytiä P (2004). Ionotropic glutamate receptor antagonists modulate cue-induced reinstatement of ethanolseeking behavior. Alcohol Clin Exp Res 28: 558-565.

Carloni S, Mazzoni E, Balduini W (2004). Caspase-3 and calpain activities after acute and repeated ethanol administration during the rat brain growth spurt. J Neurochem 89: 197-203.

Cho HI, Choi JW, Lee SM (2014). Impairment of autophagosomelysosome fusion contributes to chronic ethanol-induced liver injury. Alcohol 48: 717-725.

Creeley C, Wozniak DF, Labruyere J, Taylor GT, Olney JW (2006). Low doses of memantine disrupt memory in adult rats. J Neurosci 26: 3923-3932.

Danysz W, Essmann U, Bresink I, Wilke R (1994). Glutamate antagonists have different effects on spontaneous locomotor activity in rats. Pharmacol Biochem Behav 48: 111-118.

Evans SM, Levin FR, Brooks DJ, Garawi F (2007). A pilot doubleblind treatment trial of memantine for alcohol dependence. Alcohol Clin Exp Res 31: 775-782.

Goll DE, Thompson VF, Li H, Wei W, Cong J (2003). The calpain system. Physiol Rev 83: 731-801.

Hermann D, Weber-Fahr W, Sartorius A, Hoerst M, Frischknecht U, Tunc-Skarka N et al (2012). Translational magnetic resonance spectroscopy reveals excessive central glutamate levels during alcohol withdrawal in humans and rats. Biol Psychiatry 71: 1015-1021.

Holmes A, Spanagel R, Krystal JH (2013). Glutamatergic targets for new alcohol medications. Psychopharmacology 229: 539-554.

Hölter SM, Danysz W, Spanagel R (2000). Novel uncompetitive N-methyl-D-aspartate (NMDA)-receptor antagonist MRZ 2/579 suppresses ethanol intake in long-term ethanol-experienced rats and generalizes to ethanol cue in drug discrimination procedure. J Pharmacol Exp Ther 292: 545-552.

Hölter SM, Engelmann M, Kirschke C, Liebsch G, Landgraf R, Spanagel R (1998). Long-term ethanol self-administration 
with repeated ethanol deprivation episodes changes ethanol drinking pattern and increases anxiety-related behaviour during ethanol deprivation in rats. Behav Pharmacol 9: 41-48.

Hundt W, Danysz W, Holter SM, Spanagel R (1998). Ethanol and N-methyl-D-aspartate receptor complex interactions: a detailed drug discrimination study in the rat. Psychopharmacology 135: 44-51.

Koch H, Bespalov A, Drescher K, Franke H, Krügel U (2015). Impaired cognition after stimulation of $\mathrm{P} 2 \mathrm{Y} 1$ receptors in the rat medial prefrontal cortex. Neuropsychopharmacology 40: 305-314.

Kostowski W, Bieńkowski P (1999). Discriminative stimulus effects of ethanol: neuropharmacological characterization. Alcohol 17: 63-80.

Krupitsky EM, Neznanova O, Masalov D, Burakov AM, Didenko T, Romanova $\mathrm{T}$ et al (2007). Effect of memantine on cue-induced alcohol craving in recovering alcohol-dependent patients. Am J Psychiatry 164: 519-523.

Krystal JH, D'Souza DC, Petrakis IL, Belger A, Berman RM, Charney DS et al (1999). NMDA agonists and antagonists as probes of glutamatergic dysfunction and pharmacotherapies in neuropsychiatric disorders. Harv Rev Psychiatry 7: 125-143.

Krystal JH, Petrakis IL, Mason G, Trevisan L, D'Souza DC (2003). $\mathrm{N}$-methyl-D-aspartate glutamate receptors and alcoholism: reward, dependence, treatment, and vulnerability. Pharmacol Ther 99: 79-94.

Lovinger DM, White G, Weight FF (1989). Ethanol inhibits NMDAactivated ion current in hippocampal neurons. Science 243: 1721-1724.

Lubisch W, Beckenbach E, Bopp S, Hofmann HP, Kartal A, Kaestel C et al (2003). Benzoylalanine-derived ketoamides carrying vinylbenzyl amino residues: discovery of potent watersoluble calpain inhibitors with oral bioavailability. J Med Chem 46: 2404-2412.

Meinhardt MW, Hansson AC, Perreau-Lenz S, Bauder-Wenz C, Stählin O, Heilig M et al (2013). Rescue of infralimbic mGluR2 deficit restores control over drug-seeking behavior in alcohol dependence. J Neurosci 33: 2794-2806.

Meinhardt MW, Sommer WH (2015). Postdependent state in rats as a model for medication development in alcoholism. Addict Biol 20: $1-21$.

Nimmrich V, Bespalov A, Möller A (2012). Calpain. Encyclopedia of Signaling Molecules, Springer, New York, pp 225-228.

Nimmrich V, Szabo R, Nyakas C, Granic I, Reymann KG, Schröder UH et al (2008). Inhibition of calpain prevents N-Methyl-D-aspartate-induced degeneration of the nucleus basalis and associated behavioral dysfunction. J Pharmacol Exp Ther 327: 343-352.

Noori HR, Spanagel R, Hansson AC (2012). Neurocircuitry for modeling drug effects. Addict Biol 17: 827-864.

Nyakas C, Granic I, Halmy LG, Banerjee P, Luiten PG (2011). The basal forebrain cholinergic system in aging and dementia. Rescuing cholinergic neurons from neurotoxic amyloid- $\beta 42$ with memantine. Behav Brain Res 221: 594-603.

Rajgopal Y, Vemuri MC (2002). Calpain activation and alpha-spectrin cleavage in rat brain by ethanol. Neurosci Lett 321: 187-191.

Rammes G (2009). Neramexane: a moderate-affinity NMDA receptor channel blocker: new prospects and indications. Expert Rev Clin Pharmacol 2: 231-238.
Rimondini R, Arlinde C, Sommer W, Heilig M (2002). Long-lasting increase in voluntary ethanol consumption and transcriptional regulation in the rat brain after intermittent exposure to alcohol. FASEB J 16: 27-35.

Samson HH (1986). Initiation of ethanol reinforcement using a sucrose-substitution procedure in food- and water-sated rats. Alcohol Clin Exp Res 10: 436-442.

Sanchis-Segura C, Spanagel R (2006). Behavioural assessment of drug-reinforcement and addictive features in rodents: an overview. Addict Biol 11: 2-38.

Shaham Y, Shalev U, Lu L, De Wit H, Stewart J (2003). The reinstatement model of drug relapse: history, methodology and major findings. Psychopharmacology 168: 3-20.

Spanagel R (2009). Alcoholism: a systems approach from molecular physiology to addictive behavior. Physiol Rev 89: 649-705.

Spanagel R, Durstewitz D, Hansson A, Heinz A, Kiefer F, Köhr G et al, Addiction GWAS Resource Group (2013). A systems medicine research approach for studying alcohol addiction. Addict Biol 18: 883-896.

Spanagel R, Hölter SM (1999). Long-term alcohol self-administration with repeated alcohol deprivation phases: an animal model of alcoholism? Alcohol Alcohol 34: 231-243.

Spanagel R, Vengeliene V (2013). New pharmacological treatment strategies for relapse prevention. Curr Top Behav Neurosci 13: 583-609.

Sukhotina IA, Dravolina OA, Novitskaya Y, Zvartau EE, Danysz W, Bespalov AY (2008). Effects of mGlu1 receptor blockade on working memory, time estimation, and impulsivity in rats. Psychopharmacology 196: 211-220.

Vengeliene V, Bachteler D, Danysz W, Spanagel R (2005). The role of the NMDA receptor in alcohol relapse: a pharmacological mapping study using the alcohol deprivation effect. Neuropharmacology 48: 822-829.

Vengeliene V, Bilbao A, Molander A, Spanagel R (2008). Neuropharmacology of alcohol addiction. Br J Pharmacol 154: 299-315.

Vengeliene V, Celerier E, Chaskiel L, Penzo F, Spanagel R (2009). Compulsive alcohol drinking in rodents. Addict Biol 14: 384-396.

Vengeliene V, Heidbreder CA, Spanagel R (2007). The effects of lamotrigine on alcohol-seeking and relapse. Neuropharmacology 53: 951-957.

Vengeliene V, Leonardi-Essmann F, Sommer WH, Marston HM, Spanagel R (2010). Glycine transporter-1 blockade leads to persistently reduced relapse-like alcohol drinking in rats. Biol Psychiatry 68: 704-711.

Witzmann FA, Li J, Strother WN, McBride WJ, Hunter L, Crabb DW et al (2003). Innate differences in protein expression in the nucleus accumbens and hippocampus of inbred alcohol-preferring and -nonpreferring rats. Proteomics 3: $1335-1344$.

Zakharova E, Malyshkin A, Kashkin V, Neznanova O, Sukhotina I, Danysz W et al (2004). The NMDA receptor channel blocker memantine and opioid receptor antagonist naltrexone inhibit the saccharin deprivation effect in rats. Behavioural Pharmacology 15: 273-278.

Supplementary Information accompanies the paper on the Neuropsychopharmacology website (http://www.nature.com/npp)

Neuropsychopharmacology 